\title{
Base change and $K$-theory for GL(n)
}

\author{
Sergio Mendes and Roger Plymen
}

\begin{abstract}
Let $F$ be a nonarchimedean local field and let $G=\mathrm{GL}(n)=\mathrm{GL}(n, F)$. Let $E / F$ be a finite Galois extension. We investigate base change $E / F$ at two levels: at the level of algebraic varieties, and at the level of $K$-theory. We put special emphasis on the representations with Iwahori fixed vectors, and the tempered spectrum of GL(1) and GL(2). In this context, the prominent arithmetic invariant is the residue degree $f(E / F)$.
\end{abstract}

Mathematics Subject Classification (2000). 22E50, 46L80.

Keywords. Local field, general linear group, algebraic variety, base change, K-theory.

\section{Introduction}

The domain of definition of the classical modular forms (the upper half plane) is a homogeneous space $\mathbb{U}=\{z \in \mathbb{C}: \operatorname{Im} z>0\}$ of the reductive group $G(\mathbb{R})=$ $\operatorname{GL}(2, \mathbb{R})$,

$$
\mathrm{M}=\mathrm{GL}(2, \mathbb{R}) / \mathrm{O}(2, \mathbb{R}) \cdot Z,
$$

where $Z$ is the centre of $G(\mathbb{R})$ and $\mathrm{O}(2, \mathbb{R})$ is the orthogonal group. Each modular form admits a lift $\tilde{f}$ to the group $\operatorname{GL}(2, \mathbb{R})$ and thence to the adele group $\operatorname{GL}(2, \mathbb{A})$.

The action of $\operatorname{GL}(2, \mathbb{A})$ on $\tilde{f}$ by right translation defines a representation $\pi=\pi_{f}$ of the group $\operatorname{GL}(2, \mathbb{A})$ in the space of smooth complex-valued representations on $\operatorname{GL}(2, \mathbb{A})$, for which

$$
(\pi(h) \tilde{f})(g)=\tilde{f}(g h)
$$

for all $g, h \in \mathrm{GL}(2, \mathbb{A})$. If $\pi_{f}$ is irreducible then one has an infinite tensor product representation

$$
\pi=\bigotimes_{v} \pi_{v}
$$

where the $\pi_{v}$ are representations of the local groups $\operatorname{GL}\left(2, \mathbb{Q}_{v}\right)$ with $v=p$ or $\infty$.

Let $F$ be a local nonarchimedean field, so that $F$ is either a finite extension of $\mathbb{Q}_{p}$ or is a local function field $\mathbb{F}_{q}((x))$. The cardinality of the residue field $k_{F}$ will be denoted $q_{F}$. If $F=\mathbb{Q}_{p}$ then $q_{F}=p$. If $F=\mathbb{F}_{q}((x))$ then $q_{F}=q$. 
Now let $G=\operatorname{GL}(n)=\mathrm{GL}(n, F)$. Brodzki and Plymen [4], working directly with $L$-parameters, equipped the smooth dual of $\operatorname{GL}(n)$ with a complex structure. In the smooth dual of $\operatorname{GL}(n)$, especially important are the representations with Iwahori fixed vectors. This part of the smooth dual has the structure of the extended quotient $\left(\mathbb{C}^{\times}\right)^{n} / / S_{n}$. This is a smooth complex affine algebraic variety denoted $\mathfrak{X}_{F}$.

Let $E / F$ be a finite Galois extension of $F$. We recall that the domain of an $L$-parameter of $\operatorname{GL}(n, F)$ is the local Langlands group

$$
\mathscr{L}_{F}:=W_{F} \times \operatorname{SL}(2, \mathbb{C})
$$

where $W_{F}$ is the Weil group of $F$. Base change is defined by restriction of $L$-parameter from $\mathscr{L}_{F}$ to $\mathscr{L}_{E}$. We prove, in Section 3, that base change

$$
\mathfrak{X}_{F} \rightarrow \mathfrak{X}_{E}
$$

is a finite morphism of algebraic varieties.

An $L$-parameter $\phi$ is tempered if $\phi\left(W_{F}\right)$ is bounded [3], §10.3. Base change therefore determines a map of tempered duals. In the rest of this article, we investigate this map at the level of K-theory.

Let $G(F)=\mathrm{GL}(n, F)$. Let $C_{r}^{*}(G)$ denote the reduced $\mathrm{C}^{*}$-algebra of $G$. According to the Baum-Connes correspondence, we have a canonical isomorphism [2], [11]

$$
\mu_{F}: K_{*}^{\text {top }}\left(\beta^{1} G(F)\right) \rightarrow K_{*} C_{r}^{*}(G(F))
$$

where $\beta^{1} G(F)$ denotes the enlarged building of $G(F)$.

In noncommutative geometry, isomorphisms of $C^{*}$-algebras are too restrictive to provide a good notion of isomorphism of noncommutative spaces, and the correct notion is provided by strong Morita equivalence of $\mathrm{C}^{*}$-algebras; this point is emphasized in [13], p. 409. In the present context, the noncommutative $\mathrm{C}^{*}$-algebra $C_{r}^{*}(G(F))$ is strongly Morita equivalent to the commutative $\mathrm{C}^{*}$-algebra $C_{0}\left(\operatorname{Irr}^{t} G(F)\right)$ where $\operatorname{Irr}^{t} G(F)$ denotes the tempered dual of $G(F)$, see [15]. As a consequence of this, we have

$$
K_{*} C_{r}^{*}(G(F)) \cong K^{*} \operatorname{Irr}^{t} G(F) .
$$

This leads to the following formulation of the Baum-Connes correspondence:

$$
K_{*}^{\text {top }}\left(\beta^{1} G(F)\right) \cong K^{*} \operatorname{Irr}^{t} G(F) .
$$

This in turn leads to the following diagram

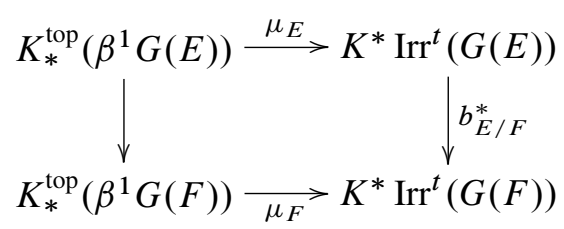


where the left-hand vertical map is the unique map which makes the diagram commutative.

In this paper we focus on the right-hand vertical map. Section 4 contains some partial results. In Sections 5 and 6, we focus on GL(1) and GL(2). We need some crucial results of Bushnell and Henniart [5]: the exact references are given in Section 6. In Section 6, the local field $F$ has characteristic 0 and $p \neq 2$. The $K$-theory map induced by unramified base change for totally ramified cuspidal representations with unitary central character is described in Theorem 6.3.

In conformity with the recent book by Bushnell and Henniart [6], we will consistently write cuspidal representation instead of supercuspidal representation.

We would like to thank Guy Henniart for his help on many occasions, and for his prompt replies to emails.

Sergio Mendes is supported by Fundação para a Ciência e Tecnologia, Terceiro Quadro Comunitário de Apoio, SFRH/BD/10161/2002.

\section{Base change formula for quasicharacters}

Let $F$ be a local nonarchimedean field. Such a field has an intrinsic norm, denoted $\bmod _{F}$ in [18], p. 4. We will write

$$
|x|_{F}=\bmod _{F}(x) \text {. }
$$

The valuation $\operatorname{val}_{F}$ is then uniquely determined by the equation

$$
|x|_{F}=q_{F}^{-\operatorname{val}_{F}(x)}
$$

where $q_{F}$ is the cardinality of the residue field $k_{F}=\mathfrak{o}_{F} / \mathfrak{p}_{F}$. Here $\mathfrak{o}_{F}$ denotes the ring of integers and $\mathfrak{p}_{F}$ its maximal ideal.

In this section, we review standard material on base change for quasicharacters. Let $E / F$ be a finite Galois extension, and let the corresponding Weil groups be denoted $W_{E}, W_{F}$. We have the standard short exact sequence

$$
1 \rightarrow I_{E} \rightarrow W_{E} \stackrel{d_{E}}{\longrightarrow} \mathbb{Z} \rightarrow 0 .
$$

Let $\operatorname{Art}_{E}^{-1}: W_{E}^{\mathrm{ab}} \cong E^{\times}$, let $\beta_{E}: W_{E} \rightarrow W_{E}^{\mathrm{ab}}$ and let

$$
\alpha_{E}=\operatorname{Art}_{E}^{-1} \circ \beta_{E}: W_{E} \rightarrow E^{\times} .
$$

Lemma 2.1. We have

$$
N_{E / F}\left(\alpha_{E}(w)\right)=\alpha_{F}(w)
$$

for all $w \in W_{E} \subset W_{F}$. 
Proof. See [17], 1.2.2.

Lemma 2.2. We have

$$
f \cdot \operatorname{val}_{E}=\operatorname{val}_{F} \circ N_{E / F} .
$$

Proof. See [18], VIII.1, p. 139.

Lemma 2.3. We have

$$
d_{E}=-\operatorname{val}_{E} \circ \alpha_{E}
$$

Proof. Let $\Phi_{E}$ denote a geometric Frobenius in $W_{E}$, as in [17, p.19]. The Weil group $W_{E}$ is the disjoint union $\sqcup I_{E} \Phi_{E}^{n}$. Then we have

$$
\begin{aligned}
\operatorname{val}_{E}\left(\alpha_{E}\left(x \Phi_{E}^{n}\right)\right) & =\operatorname{val}_{E}\left(\varpi_{E}^{-n}\right) \\
& =-n \\
& =-d_{E}\left(x \Phi_{E}^{n}\right)
\end{aligned}
$$

for all $x \in I_{E}$.

Lemma 2.4. Let $w \in W_{E} \subset W_{F}$. Then we have

$$
f \cdot d_{E}(w)=d_{F}(w) .
$$

Proof. By Lemmas 2.1, 2.2 and 2.3 we have

$$
\begin{aligned}
d_{F}(w) & =-\operatorname{val}_{F}\left(\alpha_{F}(w)\right) \\
& =-\operatorname{val}_{F}\left(N_{E / F}\left(\alpha_{E}(w)\right)\right. \\
& =-f \cdot \operatorname{val}_{E}\left(\alpha_{E}(w)\right) \\
& =f \cdot d_{E}(w) .
\end{aligned}
$$

Now an unramified quasicharacter $\psi$ of $W_{E}$ is given by the following simple formula

$$
\psi(w)=z^{d_{E}(w)}
$$

where $z \in \mathbb{C}^{\times}$. The base change formula for a quasicharacter $\chi$ of $W_{F}$ is given by

$$
b_{E / F}(\chi)=\chi_{\mid W_{E}} .
$$

Lemma 2.5. Under base change we have

$$
b_{E / F}(\psi)(w)=\left(z^{f}\right)^{d_{E}(w)}
$$

for all $w \in W_{E}$. 
Proof. By Lemma 2.4 we have

$$
\begin{aligned}
b_{E / F}(\psi)(w) & =z^{d_{F}(w)} \\
& =z^{f \cdot d_{E}(w)} \\
& =\left(z^{f}\right)^{d_{E}(w)} .
\end{aligned}
$$

We can remember this result with the (informal) equation

$$
z_{E / F}=z^{f} \text {. }
$$

\section{Representations with Iwahori fixed vectors}

Let $\Psi\left(W_{F}\right)$ denote the group of unramified quasicharacters of $W_{F}$. Then we have

$$
\Psi\left(W_{F}\right) \cong \mathbb{C}^{\times}, \quad \psi \mapsto \psi\left(\varpi_{F}\right) .
$$

Let $\mathscr{L}_{F}$ denote the local Langlands group:

$$
\mathscr{L}_{F}:=W_{F} \times \operatorname{SL}(2, \mathbb{C}) .
$$

A Langlands parameter (or $L$-parameter) is a continuous homomorphism

$$
\phi: \mathscr{L}_{F} \rightarrow \operatorname{GL}(n, \mathbb{C})
$$

(GL $(n, \mathbb{C})$ is given the discrete topology) such that $\phi\left(\Phi_{F}\right)$ is semisimple, where $\Phi_{F}$ is a geometric Frobenius in $W_{F}$. Two Langlands parameters are equivalent if they are conjugate under $\operatorname{GL}(n, \mathbb{C})$. The set of equivalence classes of Langlands parameters is denoted $\Phi(\mathrm{GL}(n))$.

We will use the local Langlands correspondence for GL(n) [12], [9], [10]:

$$
\pi_{F}: \Phi(\operatorname{GL}(n)) \rightarrow \operatorname{Irr}(\mathrm{GL}(n)) .
$$

Consider first the single $L$-parameter

$$
\phi=1 \otimes \tau\left(j_{1}\right) \oplus \cdots \oplus 1 \otimes \tau\left(j_{k}\right)
$$

where $\tau(j)$ is the $j$-dimensional complex representation of $\operatorname{SL}(2, \mathbb{C})$, and $j_{1}+\cdots$ $+j_{k}=n$. We define the orbit of $\phi$ as follows:

$$
\mathcal{O}(\phi)=\left\{\psi_{1} \otimes \tau\left(j_{1}\right) \oplus \cdots \oplus \psi_{k} \otimes \tau\left(j_{k}\right): \psi_{r} \in \Psi\left(W_{F}\right), 1 \leq r \leq k\right\} / \sim
$$

where $\sim$ denotes the equivalence relation of conjugacy in $\operatorname{GL}(n, \mathbb{C})$.

In the local Langlands correspondence, these $L$-parameters correspond precisely to the irreducible smooth representations of $\mathrm{GL}(n)$ which admit Iwahori fixed vectors. 
Each partition $j_{1}+\cdots+j_{k}=n$ determines an orbit. The disjoint union of the orbits, one for each partition of $n$, creates a complex affine algebraic variety with finitely many irreducible components. This variety is smooth [4]. This variety admits a simple description as an extended quotient, as we now proceed to explain.

Let $\Gamma$ be a finite group and $X$ a topological space. Suppose that $\Gamma$ acts on $X$ as homeomorphisms. Define

$$
\tilde{X}=\{(\gamma, x) \in \Gamma \times X: \gamma x=x\}
$$

and

$$
g \cdot(\gamma, x)=\left(g \gamma g^{-1}, g x\right) \text {, }
$$

for all $g \in \Gamma$ and $(\gamma, x) \in \tilde{X}$. Since $\left(g \gamma g^{-1}\right)(g x)=g(\gamma x)=g x, \Gamma$ acts on $\tilde{X}$.

Definition 3.1. The extended quotient $X / / \Gamma$ associated to the action of $\Gamma$ on $X$ is the quotient space $\tilde{X} / \Gamma$.

If $\gamma \in \Gamma$, let $X^{\gamma}$ denote fixed set

$$
X^{\gamma}=\{x \in X: \gamma x=x\}
$$

and let $Z_{\gamma}$ denote the centralizer of $\gamma$ in $\Gamma$. Then the extended quotient is the disjoint union

$$
X / / \Gamma=\sqcup X^{\gamma} / Z_{\gamma}
$$

where one $\gamma$ is chosen in each $\Gamma$-conjugacy class.

Let $X=\left(\mathbb{C}^{\times}\right)^{n}$ be the complex torus of dimension $n$. The symmetric group $\Gamma=S_{n}$ acts on $X$ by permuting the coordinates. First, we form the ordinary quotient:

$$
\operatorname{Sym}^{n}\left(\mathbb{C}^{\times}\right):=\left(\mathbb{C}^{\times}\right)^{n} / S_{n},
$$

the $n$-fold symmetric product of $\mathbb{C}^{\times}$.

Next, we form the extended quotient $\left(\mathbb{C}^{\times}\right)^{n} / / S_{n}$. The conjugacy class of $\gamma \in S_{n}$ determines a partition of $n$. Let the distinct parts of the partition be $n_{1}, \ldots, n_{l}$ with $n_{i}$ repeated $r_{i}$ times so that

$$
r_{1} n_{1}+\cdots+r_{l} n_{l}=n .
$$

Let

$$
z_{j}=\psi_{j}\left(\varpi_{F}\right) .
$$

The map

$$
\psi_{1} \otimes \tau\left(n_{1}\right) \oplus \cdots \oplus \psi_{r_{1}+\cdots+r_{l}} \otimes \tau\left(n_{l}\right) \mapsto\left(z_{1}, \ldots, z_{r_{1}+\cdots+r_{l}}\right)
$$

determines a bijection

$$
\mathcal{O}(\phi) \sim \operatorname{Sym}^{r_{1}}\left(\mathbb{C}^{\times}\right) \times \cdots \times \operatorname{Sym}^{r_{l}}\left(\mathbb{C}^{\times}\right)=X^{\gamma} / Z(\gamma) .
$$


With a mild abuse of notation, we will write the $L$-parameter

$$
\phi=\psi_{1} \otimes \tau\left(j_{1}\right) \oplus \cdots \oplus \psi_{k} \otimes \tau\left(j_{k}\right)
$$

as

$$
z_{1} \cdot \tau\left(j_{1}\right) \oplus \cdots \oplus z_{k} \cdot \tau\left(j_{k}\right)
$$

After base change $E / F$ this $L$-parameter becomes

$$
z_{1}^{f} \cdot \tau\left(j_{1}\right) \oplus \cdots \oplus z_{k}^{f} \cdot \tau\left(j_{k}\right) .
$$

Example 3.2. We illustrate this result for GL(4), by computing the $L$-parameters, the respective orbits and the extended quotient. For each item, we list the partition, the $L$-parameter and the orbit:

- $4+0, \phi=1 \otimes \tau(4), \mathcal{O}(\phi) \cong \mathbb{C}^{\times}$

- $3+1, \phi=1 \otimes \tau(3) \oplus 1 \otimes 1, \mathcal{O}(\phi) \cong\left(\mathbb{C}^{\times}\right)^{2}$

- $2+2, \phi=1 \otimes \tau(2) \oplus 1 \otimes \tau(2), \mathcal{O}(\phi) \cong \operatorname{Sym}^{2}\left(\mathbb{C}^{\times}\right)$

- $2+1+1, \phi=1 \otimes \tau(2) \oplus 1 \otimes 1 \oplus 1 \otimes 1, \mathcal{O}(\phi) \cong \mathbb{C}^{\times} \times \operatorname{Sym}^{2}\left(\mathbb{C}^{\times}\right)$

- $1+1+1+1, \phi=1 \otimes 1 \oplus 1 \otimes 1 \oplus 1 \otimes 1 \oplus 1 \otimes 1, \mathcal{O}(\phi) \cong \operatorname{Sym}^{4}\left(\mathbb{C}^{\times}\right)$

and the extended quotient is

$$
\left(\mathbb{C}^{\times}\right)^{4} / / S_{4}=\mathbb{C}^{\times} \sqcup\left(\mathbb{C}^{\times}\right)^{2} \sqcup \operatorname{Sym}^{2}\left(\mathbb{C}^{\times}\right) \sqcup \mathbb{C}^{\times} \times \operatorname{Sym}^{2}\left(\mathbb{C}^{\times}\right) \sqcup \operatorname{Sym}^{4}\left(\mathbb{C}^{\times}\right) .
$$

Theorem 3.3. Let $\mathfrak{X}_{F}$ be that part of the smooth dual of $\mathrm{GL}(n, F)$ comprising all representations which admit Iwahori fixed vectors. Then $\mathfrak{X}_{F}$ is a smooth complex affine algebraic variety, and in fact has the structure of extended quotient:

$$
\mathfrak{X}_{F}=\left(\mathbb{C}^{\times}\right)^{n} / / S_{n}
$$

Let $E / F$ be a finite Galois extension. Then base change

$$
\mathfrak{X}_{F} \rightarrow \mathfrak{X}_{E}
$$

is a finite morphism of algebraic varieties. Explicitly, if $z_{1}, \ldots, z_{r}$ are typical coordinates on $\mathfrak{X}_{F}$, then base change is given by

$$
\left(z_{1}, \ldots, z_{r}\right) \mapsto\left(z_{1}^{f}, \ldots, z_{r}^{f}\right) .
$$

Proof. A regular map $f: X \rightarrow Y$ of affine varieties is finite if $\mathbb{C}[X]$ is integral over $\mathbb{C}[Y]$, i.e., if the pullback $f^{\sharp}: \mathbb{C}[Y] \rightarrow \mathbb{C}[X]$ makes $\mathbb{C}[X]$ a finitely generated $\mathbb{C}[Y]$-module. The map $\mathfrak{X}_{F} \rightarrow \mathfrak{X}_{E}$ is regular. 
Each irreducible component in the algebraic variety $\mathfrak{X}_{F}$ is a product of symmetric products. Denote a typical symmetric product by $\mathfrak{S}_{F}$. The coordinate ring of each symmetric product $\mathfrak{S}_{F}$ is of the form

$$
\mathbb{C}\left[t_{1}, \ldots, t_{r}, t_{1}^{-1}, \ldots, t_{r}^{-1}\right]^{S_{r}}
$$

the ring of invariant Laurent polynomials. The pullback is

$$
\mathbb{C}\left[\Im_{E}\right] \rightarrow \mathbb{C}\left[\Im_{F}\right], \quad t_{i} \mapsto t_{i}^{f} .
$$

Since $\mathbb{C}\left[\mathfrak{S}_{F}\right]$ is finitely generated as a $\mathbb{C}\left[\subseteq_{E}\right]$-module, the base change map is finite.

Example 3.4. The unramified twists of the Steinberg representation of GL(n).

These representations correspond, in the local Langlands correspondence, to the orbit of the single $L$-parameter $1 \otimes \tau(n)$. This creates an irreducible curve $\mathfrak{Y}_{F}$ in the smooth dual of $\mathrm{GL}(n, F)$, in fact $\mathfrak{Y}_{F} \cong \mathbb{C}^{\times}$. Base change $E / F$ is as follows:

$$
\mathfrak{Y}_{F} \rightarrow \mathfrak{Y}_{E}, \quad z \mapsto z^{f} .
$$

Example 3.5. The spherical Hecke algebra.

Let $K=\operatorname{GL}\left(n, \mathfrak{o}_{F}\right)$ and denote by $\mathscr{H}(G / / K)$ the convolution algebra of all complex-valued, compactly-supported functions on $G$ such that $f\left(k_{1} x k_{2}\right)=f(x)$ for all $k_{1}, k_{2}$ in $K$. Then $\mathscr{H}(G / / K)$ is called the spherical Hecke algebra. It is a commutative unital $\mathbb{C}$-algebra.

Start with the single $L$-parameter

$$
\phi=1 \otimes 1 \oplus \cdots \oplus 1 \otimes 1
$$

and let $\mathcal{O}(\phi)$ denote the orbit of $\phi$. We have

$$
\mathcal{O}(\phi)=\left\{\psi_{1} \otimes 1 \oplus \cdots \oplus \psi_{n} \otimes 1\right\} / \sim
$$

with $\psi_{j}$ an unramified quasicharacter of $W_{F}, 1 \leq j \leq n$. Let $T$ be the standard maximal torus of GL(n), and let ${ }^{L} T$ be the standard maximal torus in the Langlands dual ${ }^{L} G$ :

$$
{ }^{L} T \subset{ }^{L} G=\operatorname{GL}(n, \mathbb{C}) .
$$

Let $W=S_{n}$ the symmetric group on $n$ letters. Then we have

$$
\mathcal{O}(\phi) \cong \mathbb{C}\left[{ }^{L} T / W\right]=\operatorname{Sym}^{n}\left(\mathbb{C}^{\times}\right) .
$$


As a special case of the above, base change $E / F$ induces the following finite morphism of algebraic varieties:

$$
\operatorname{Sym}^{n}\left(\mathbb{C}^{\times}\right) \rightarrow \operatorname{Sym}^{n}\left(\mathbb{C}^{\times}\right), \quad\left(z_{1}, \ldots, z_{n}\right) \mapsto\left(z_{1}^{f}, \ldots, z_{n}^{f}\right) .
$$

In view of the Satake isomorphism [7]

$$
\mathscr{H}(G / / K) \cong \mathbb{C}\left[{ }^{L} T / W\right]
$$

we can interpret base change as an explicit morphism of unital $\mathbb{C}$-algebras. This recovers (and generalizes) the result in [1], p. 37, for we do not require the extension $E / F$ to be either unramified or cyclic.

\section{4. $K$-theory computations}

In this section we compute the $K$-theory map for two examples: the unitary twists of the Steinberg representation of $\operatorname{GL}(n)$, and certain connected components in the unitary principal series of $\operatorname{GL}(n)$.

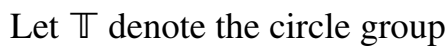

$$
\mathbb{T}=\{z \in \mathbb{C}:|z|=1\}
$$

and let $\Psi^{t}\left(W_{F}\right)$ denote the group of unramified unitary characters of $W_{F}$. Then we have

$$
\Psi^{t}\left(W_{F}\right) \cong \mathbb{T}, \quad \psi \mapsto \psi\left(\varpi_{F}\right)
$$

where $\varpi_{F}$ is a uniformizer in $F$.

Consider first the single $L$-parameter

$$
\phi=\rho \otimes \tau\left(j_{1}\right) \oplus \cdots \oplus \rho \otimes \tau\left(j_{k}\right)
$$

In this formula, $\rho$ is an irreducible representation of $W_{F}, \tau(j)$ is the $j$-dimensional complex representation of $\operatorname{SL}(2, \mathbb{C})$, and $j_{1}+\cdots+j_{k}=n$. We define the compact orbit of $\phi$ as follows:

$$
\mathcal{O}^{t}(\phi)=\left\{\bigoplus_{r=1}^{k} \psi_{r} \otimes \rho \otimes \tau\left(j_{r}\right): \psi_{r} \in \Psi^{t}\left(W_{F}\right), 1 \leq r \leq k\right\} / \sim
$$

where, as before, $\sim$ denotes the equivalence relation of conjugacy in $\operatorname{GL}(n, \mathbb{C})$.

The Steinberg representation $\mathrm{St}_{G}$ has $L$-parameter $1 \otimes \tau(n)$. 
Theorem 4.1. Let

$$
\phi=1 \otimes \tau(n)
$$

and let $\mathcal{O}^{t}(\phi)$ be the compact orbit of $\phi$. Then we have

$$
B C: \mathbb{T} \rightarrow \mathbb{T}, \quad z \mapsto z^{f} .
$$

(i) This map has degree $f$, and so at the level of the $K$-theory group $K^{1}, B C$ induces the map

$$
\mathbb{Z} \rightarrow \mathbb{Z}, \quad \alpha_{1} \mapsto f \cdot \alpha_{1}
$$

of multiplication by the residue degree $f$. Here, $\alpha_{1}$ denotes a generator of $K^{1}(\mathbb{T}) \cong \mathbb{Z}$.

(ii) At the level of the $K$-theory group $K^{0}, B C$ induces the identity map

$$
\mathbb{Z} \rightarrow \mathbb{Z}, \quad \alpha_{0} \mapsto \alpha_{0},
$$

where $\alpha_{0}$ denotes a generator of $K^{0}(\mathbb{T}) \cong \mathbb{Z}$.

Proof. (i) Since the map has degree $f$ the result follows.

(ii) This is because $\alpha_{0}$ is the trivial bundle of rank 1 over $\mathbb{T}$.

Next we define the $L$-parameter $\phi$ as follows:

$$
\phi=\rho \otimes 1 \oplus \cdots \oplus \rho \otimes 1
$$

where $\rho$ is a unitary character of $W_{F}$. The unitary characters of $W_{F}$ factor through $F^{\times}$and we have

$$
F^{\times} \cong\left\langle\varpi_{F}\right\rangle \times u_{F} .
$$

We will take $\rho$ to be trivial on $\left\langle\varpi_{F}\right\rangle$, and then regard $\rho$ as a unitary character of $u_{F}$. The group $U_{F}$ admits countably many such characters $\rho$.

In this case the compact orbit is the $n$-fold symmetric product of the circle $\mathbb{T}$ :

$$
\mathcal{O}^{t}(\phi) \cong \mathcal{O}^{t}(B C(\phi)) \cong \mathbb{T}^{n} / S_{n}
$$

Lemma 4.2. The symmetric product $\mathbb{T}^{n} / S_{n}$ has the homotopy type of a circle.

Proof. Send the unordered $n$-tuple $[z]=\left[z_{1}, \ldots, z_{n}\right]$ to the unique polynomial with roots $z_{1}, \ldots, z_{n}$ and leading coefficient 1

$$
\left[z_{1}, \ldots, z_{n}\right] \mapsto z^{n}+a_{n-1} z^{n-1}+\cdots+a_{1} z+a_{0}, \quad a_{0} \neq 0 .
$$

We have then

$$
\operatorname{Sym}^{n}\left(\mathbb{C}^{\times}\right) \cong\left\{z^{n}+a_{n-1} z^{n-1}+\cdots+a_{1} z+a_{0}: a_{0} \neq 0\right\} \sim_{h} \mathbb{C}^{\times},
$$


since the space of coefficients $a_{n-1}, \ldots, a_{1}$ is contractible. Hence

$$
\operatorname{Sym}^{n}(\mathbb{T}) \sim_{h} \mathbb{T}
$$

via the map which sends $\left[z_{1}, \ldots, z_{n}\right]$ to the product $z_{1} \ldots z_{n}$.

We recall the local Langlands correspondence

$$
\pi_{F}: \Phi(\operatorname{GL}(n)) \rightarrow \operatorname{Irr} \operatorname{GL}(n)
$$

Let $t=\operatorname{diag}\left(x_{1}, \ldots, x_{n}\right)$ be a diagonal element in the standard maximal torus $T$ of $\operatorname{GL}(n)$. Then

$$
\sigma: t \mapsto \pi_{F}(\rho)\left(x_{1} \ldots x_{n}\right)
$$

is a unitary character of $T$. Let $\chi$ be an unramified unitary character of $T$, and form the induced representation

$$
\operatorname{Ind}_{T U}^{G}(\chi \otimes \sigma)
$$

This is an irreducible unitary representation of $G$. When we let $\chi$ vary over all unramified unitary characters of $T$, we obtain a subset of the unitary dual of $G$. This subset has the structure of $n$-fold symmetric product of $\mathbb{T}$.

Since $U_{F}$ admits countably many unitary characters, the unitary dual of $G$ contains countably many subspaces (in the Fell topology), each with the structure $\operatorname{Sym}^{n}(\mathbb{T})$. We are concerned with the effect of base change $E / F$ on each of these compact spaces.

Theorem 4.3. Let $\mathbb{T}^{n} / S_{n}$ denote one of the compact subspaces of the unitary principal series of $\mathrm{GL}(n)$ currently under discussion. Then we have

$$
B C: \mathbb{T}^{n} / S_{n} \rightarrow \mathbb{T}^{n} / S_{n}, \quad\left(z_{1}, \ldots, z_{n}\right) \mapsto\left(z_{1}^{f}, \ldots, z_{n}^{f}\right) .
$$

(i) At the level of the $K$-theory group $K^{1}, B C$ induces the map

$$
\mathbb{Z} \rightarrow \mathbb{Z}, \quad \alpha_{1} \mapsto f \cdot \alpha_{1}
$$

of multiplication by $f$, where $f$ is the residue degree and $\alpha_{1}$ denotes a generator of $K^{1}(\mathbb{T})=\mathbb{Z}$.

(ii) At the level of the $K$-theory group $K^{0}, B C$ induces the identity map

$$
\mathbb{Z} \rightarrow \mathbb{Z}, \quad \alpha_{0} \mapsto \alpha_{0},
$$

where $\alpha_{0}$ denotes a generator of $K^{0}(\mathbb{\top})=\mathbb{Z}$. 
Proof. From Lemma (4.2) we have a commutative diagram:

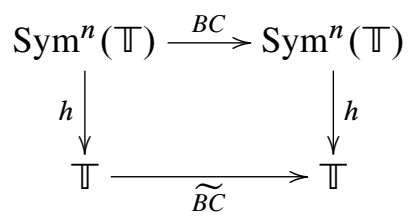

Here, $B C\left(z_{1}, \ldots, z_{n}\right)=\left(z_{1}^{f}, \ldots, z_{n}^{f}\right), h$ is the homotopy map $h\left(\left[z_{1}, \ldots, z_{n}\right]\right)=$ $z_{1} \ldots z_{n}$ and $\widetilde{B C}$ is the map $z \mapsto z^{f}$. Since

$$
\left(z_{1} \ldots z_{n}\right)^{f}=z_{1}^{f} \ldots z_{n}^{f}
$$

we have $K^{j}(B C)=K^{j}(\widetilde{B C})$. But $\widetilde{B C}$ is a map of degree $f$. Therefore,

$$
K^{1}(B C)\left(\alpha_{1}\right)=f . \alpha_{1} \text { and } K^{0}(B C)\left(\alpha_{0}\right)=\alpha_{0}
$$

where $\alpha_{1}$ is a generator of $K^{1}(\mathbb{T})=\mathbb{Z}$ and $\alpha_{0}$ is a generator of $K^{0}(\mathbb{\mathbb { T }})=\mathbb{Z}$.

\section{Base change and $K$-theory for $\operatorname{GL}(1, F)$}

So far we have considered base change as a map of compact orbits. Now we want to describe base change as a map of the locally compact Hausdorff spaces

$$
B C: \mathcal{A}_{1}^{t}(F) \rightarrow \mathcal{A}_{1}^{t}(E)
$$

where $\mathcal{A}_{1}^{t}(F)$ denotes $\operatorname{Irr}^{t} \operatorname{GL}(1, F)$. From now on we will change notation and we denote the tempered dual $\operatorname{Irr}^{t} \operatorname{GL}(n, F)$ by $\mathcal{A}_{n}^{t}(F)$.

To study the effect of base change on $K$-theory groups we explicitly compute the functorial base change map $K^{j}(B C)$. We will use $K$-theory with compact supports and in particular we will prove that $B C$ is a proper map.

Let $\chi=||_{F}^{S} \chi_{0}$ be a character of $F^{\times}$, where $\chi_{0}$ is the restriction of $\chi$ to ${ }^{\times} F$. We will write from now on $\chi=z^{v_{F}^{(\cdot)}} \chi_{0}$ (since $|x|_{F}^{s}=q_{F}^{-s v_{F}(x)}$ this is simply a change of variables $q_{F}^{-s} \in \mathbb{T} \mapsto z \in \mathbb{T}$ ). We also denote the group of units $\mathfrak{o}_{F}^{\times}$by $U_{F}$.

If $\chi_{0}$ is a character of $U_{F}$ then $\chi_{0}$ is trivial on some $U_{F}^{m}$. The least $m$ such that $\chi_{0}=\chi_{\mid u_{F}}$ is trivial on $U_{F}^{m}$ is called the conductor of $\chi$ and is denoted $c(\chi)$. Note that $\chi_{0}=\chi_{\mid} u_{F}$ can be thought as a character of the finite cyclic group $u_{F} / u_{F}^{c(\chi)}$.

It is well known that the parameters $(z, c(\chi)) \in \mathbb{T} \times \mathbb{N}_{0}$ do not completely determine the character $\chi$. There is a group isomorphism

$$
u_{F} / u_{F}^{m} \cong u_{F} / u_{F}^{1} \times u_{F}^{1} / u_{F}^{2} \times \cdots \times u_{F}^{m-1} / u_{F}^{m} .
$$

Now $U_{F} / u_{F}^{1} \cong k_{F}^{\times}$while $u_{F}^{i-1} / U_{F}^{i} \cong k_{F}$ for $i \geq 1$ [8], Proposition 5.4. Note that $k_{F}^{\times}$is interpreted as a multiplicative group while $\bar{k}_{F}$ is interpreted as an additive 
group. Since $k_{F}$ has order $q_{F}$ and $k_{F}^{\times}$has order $q_{F}-1$ it follows that $U_{F} / u_{F}^{m}$ is a finite cyclic group of order $\left(q_{F}-1\right) q_{F}^{m-1}$ and $\overline{U_{F} / U_{F}^{m}} \cong U_{F} / U_{F}^{m}$ is also finite with the same order.

We have [8], Lemma 3.4,

$$
F^{\times} \cong\left\langle\varpi_{F}\right\rangle \times U_{F}
$$

where $\varpi_{F}$ is a uniformizer if $F$. It follows that

$$
\mathcal{A}_{1}^{t}(F) \cong \mathbb{T} \times \widehat{u_{F}} .
$$

We will keep in mind the following enumeration of the countable set $\widehat{u_{F}}$ : to each natural number $n$ we attach the finite set of all characters $\chi \in \widehat{u_{F}}$ for which $c(\chi)=n$. This enumeration is not canonical.

Proposition 5.1 ([18], Proposition 5, p. 143). The norm map $N_{E / F}: E^{\times} \rightarrow F^{\times}$ determines an open morphism of $E^{\times}$onto an open subgroup of $F^{\times}$.

It follows that there exist $m, n \in \mathbb{N}_{0}$ such that

$$
N_{E / F}\left(U_{E}^{n}\right)=U_{F}^{m} .
$$

A natural question is how to relate the indexes $n$ and $m$. For unramified extensions we have the following result.

Proposition 5.2 ([16], Proposition 1, p. 81). Let E/F be a finite, separable, unramified extension. Suppose that $k_{F}$ is finite. Then

$$
N_{E / F}\left(U_{E}^{n}\right)=U_{F}^{n} \text { for all } n \geq 0 .
$$

Apart from unramified extensions, we will consider the cases when the extension is tamely ramified and totally ramified, since the ramification theory is simpler. The case of wildly ramified extensions is more subtle and will not be considered. We now recall some results about ramification theory. Let $E / F$ be a finite Galois extension and $G=\operatorname{Gal}(E / F)$. Put

$$
G_{i}=\left\{\sigma \in G: \sigma x-x \in \mathfrak{p}_{E}^{i+1} \text { for all } x \in \mathfrak{o}_{E}\right\}, \quad i \geq-1 .
$$

The group $G_{i}$ is called the $i$-th ramification group of the extension $E / F$. Altogether, they form a decreasing sequence of subgroups

$$
G_{-1}=G \supset G_{0} \supset G_{1} \supset \cdots \supset G_{i} \supset G_{i+1} \supset \cdots .
$$

Denote by $F_{0} / F$ the maximal unramified subextension of $F$ in $E / F$. Note that $F_{0}$ is the intersection of $E$ with the maximal unramified subextension of $\bar{F} / F$, denoted by 
$F^{u r}$, where $\bar{F}$ is a fixed algebraic closure of $F$. The subgroup $I_{E / F}=\operatorname{Gal}\left(E / F_{0}\right)$ is called the inertia subgroup of $\operatorname{Gal}(E / F)$ and we have the identification $G_{0}=$ $I_{E / F}$ [16], Proposition 1, p. 62. It follows that $G / G_{0}=\operatorname{Gal}\left(k_{E} / k_{F}\right)$ and $E / F$ is unramified if and only if $G_{0}=\{1\}$.

The quotient group $G_{0} / G_{1}$ is cyclic and has order prime to the characteristic residue of $E$ [16], Corollary 1, p. 67. Therefore, the tame ramification is given by the groups $G_{0}$ and $G_{1}$. In particular, the extension $E / F$ is tamely ramified if and only if $G_{1}=\{1\}$. These results can be summarized in the following diagram.

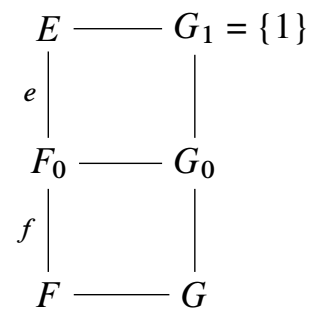

To every finite (separable) extension $E / F$ of local fields we associate a real function [16], §3, p. 73,

$$
\varphi_{E / F}(u)=\int_{0}^{u} \frac{d t}{\left(G_{0}: G_{t}\right)} \quad \text { for all } u \in[-1,+\infty[.
$$

Remark 5.3. This is simply extending the definition of the filtration (4) indexed by a finite discrete parameter to a decreasing filtration $\left\{G_{t}\right\}_{t \geq-1}$ with a continuous parameter. If $i-1<t \leq i$ then we define $G_{t}=G_{i} \cdot \varphi_{E / F}$ is a step function and is a homeomorphism of the interval $[-1,+\infty[$ into itself.

The inverse $\psi_{E / F}=\varphi_{E / F}^{-1}$ is called the Hasse-Herbrand function. We collect some properties of $\psi_{E / F}$ [16], Proposition 13, p. 73:

(i) The function $\psi_{E / F}$ is continuous, piecewise linear, increasing and convex.

(ii) $\psi_{E / F}(0)=0$.

(iii) If $v$ is an integer, then $\psi_{E / F}(v)$ is also an integer.

Example 5.4. Suppose $E / F$ is unramified. Then $G_{0}=\{1\}$ and we have

$$
\varphi_{E / F}(u)=\int_{0}^{u} \frac{d t}{\left(G_{0}: G_{t}\right)}=u .
$$

Therefore, $\psi_{E / F}(x)=x$.

Now let $E / F$ be a tame extension. Then $\left|G_{0}\right|=e, G_{1}=\{1\}$, and we have

$$
\varphi_{E / F}(u)=\int_{0}^{u} \frac{d t}{\left(G_{0}: G_{1}\right)}=\int_{0}^{u} \frac{\left|G_{1}\right|}{\left|G_{0}\right|} d t=\int_{0}^{u} \frac{d t}{e}=u / e .
$$

It follows that $\psi_{E / F}(x)=e x$. 
Example 5.5 ([16], p. 83). If $E / F$ is cyclic, totally ramified with prime degree $p$, then

$$
\psi(x)= \begin{cases}x, & x \leq t, \\ t+p(x-t), & x \geq t,\end{cases}
$$

where $t$ is such that $G_{t} \neq\{1\}$ and $\{1\}=G_{t+1}=G_{t+2}=\cdots$.

Proposition 5.6 ([16], Corollary 4, p. 93). Assume that E/F is a Galois extension, totally ramified. Let $v$ be a non-negative number and suppose that $G_{\psi(v)}=\{1\}$. Then

$$
N_{E / F}\left(u_{E}^{\psi(v)}\right)=u_{F}^{\nu}
$$

We now deduce a similar result for Galois tamely ramified extensions.

Proposition 5.7. Let $E / F$ be a tamely ramified extension. Then

$$
N_{E / F}\left(U_{E}^{\psi(v)}\right)=U_{F}^{v}
$$

for all non-negative real numbers $v$.

Proof. Let $F_{0} / F$ be the maximal unramified subextension of $F$ in $E / F$. We have a tower of fields $F \subset F_{0} \subset E$. Then $E / F_{0}$ is a totally tamely ramified extension. Since $G_{1}=\{1\}$, we also have $G_{\psi(v)}=\{1\}$, for all $v \geq 0$, where $\psi$ denotes the Hasse-Herbrand function $\psi_{E / F}$.

The conditions of Proposition 5.6 are satisfied and we have

$$
N_{E / F_{0}}\left(U_{E}^{\psi(v)}\right)=U_{F_{0}}^{\nu}
$$

Since $F_{0} / F$ is unramified, it follows from Proposition 5.2 that

$$
N_{F_{0} / F}\left(U_{F_{0}}^{v}\right)=U_{F}^{v}
$$

Finally, by transitivity of the norm map we have

$$
N_{E / F}\left(U_{E}^{\psi(v)}\right)=N_{F_{0} / F}\left(N_{E / F_{0}}\left(U_{E}^{\psi(v)}\right)\right)=N_{F_{0} / F}\left(U_{F_{0}}^{v}\right)=U_{F}^{v} .
$$

\section{Base change for GL(1) on the admissible side}

Let $E / F$ be a finite Galois extension and let $W_{E} \hookrightarrow W_{F}$ denote the inclusion of Weil groups. Langlands functoriality predicts the existence a commutative diagram

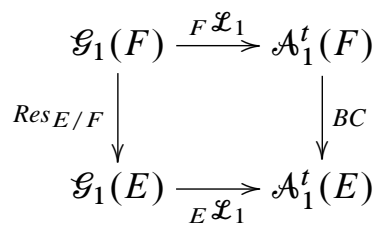


where $\mathscr{E}_{1}(F)$ (resp. $\mathscr{E}_{1}(E)$ ) is the group of characters of $W_{F}$ (resp. $W_{E}$ ) and $B C$ is the base change map. On the admissible side base change is given by

$$
\begin{aligned}
\mathcal{A}_{1}^{t}(F) & \rightarrow \mathcal{A}_{1}^{t}(E), \\
\chi_{F} & \mapsto \chi_{F} \circ N_{E / F} .
\end{aligned}
$$

Lemma 5.8. Let $\chi_{F}$ be a character of $F^{\times}$with conductor $c\left(\chi_{F}\right)$ and consider the character $\chi_{E}:=\chi_{F} \circ N_{E / F}$ of $E^{\times}$. Suppose that we have

$$
N_{E / F}\left(U_{E}^{n}\right)=U_{F}^{c\left(\chi_{F}\right)} .
$$

Then $n$ is the conductor $c\left(\chi_{E}\right)$ of $\chi_{E}$.

Proof. We have

$$
\chi_{E}\left(U_{E}^{n}\right)=\chi_{F} \circ N_{E / F}\left(U_{E}^{n}\right)=\chi_{F}\left(u_{F}^{c\left(\chi_{F}\right)}\right)=1 .
$$

Let $r$ be any integer such that $0<r<n$. Then

$$
\begin{aligned}
U_{E}^{n} & \subset U_{E}^{r}, \\
N_{E / F}\left(\mathcal{U}_{E}^{n}\right) & \subset N_{E / F}\left(\mathcal{U}_{E}^{r}\right), \\
U_{F}^{c\left(\chi_{F}\right)} & \subset U_{F}^{s},
\end{aligned}
$$

with $c\left(\chi_{F}\right)>s$. Then

$$
\chi_{F} \circ N_{E / F}\left(\mathcal{U}_{E}^{r}\right)=\chi_{F}\left(\mathcal{U}_{F}^{s}\right) \neq 1,
$$

since $c\left(\chi_{F}\right)$ is the least integer with this property. Therefore $n=c\left(\chi_{E}\right)$.

We may now describe base change as a map of topological spaces. The unitary dual $\mathcal{A}_{1}^{t}(F)$ (resp. $\left.\mathcal{A}_{1}^{t}(E)\right)$ is a disjoint union of countably many circles, parametrized by characters $\chi \in \widehat{U_{F}}$ (resp. $\eta \in \widehat{U_{F}}$ ):

$$
\mathcal{A}_{1}^{t}(F) \cong \sqcup \mathbb{T}_{\chi}, \quad \mathcal{A}_{1}^{t}(E) \cong \sqcup \mathbb{T}_{\eta} .
$$

We recall that $\chi_{E}=\chi_{F} \circ N_{E / F}$ and $c\left(\chi_{E}\right)$ is the unique integer such that

$$
N_{E / F}\left(\mathcal{U}_{E}^{c\left(\chi_{E}\right)}\right)=\mathcal{U}_{F}^{c\left(\chi_{F}\right)} .
$$

Theorem 5.9. Let $E / F$ be unramified, tamely ramified or totally ramified (in the last case we also require $E / F$ to be cyclic). Then we have:

(1) Base change is a proper map. 
(2) When we restrict base change to one circle, we get the following:

$$
B C: \mathbb{T}_{\chi_{F}} \rightarrow \mathbb{T}_{\chi_{E}}, \quad z \mapsto z^{f}
$$

with $c\left(\chi_{E}\right)=\psi_{E / F}\left(c\left(\chi_{F}\right)\right)$.

Proof. (1) Base change maps each circle into another circle. Let $K$ be a closed arc in $\mathbb{T}_{\eta}$, and let $\eta=\chi_{E}$. Then we may write

$$
K=\left\{e^{i \theta} \in \mathbb{T}_{\eta}: \theta_{0} \leq \theta \leq \theta_{1}, \theta \in[0,2 \pi]\right\} .
$$

The pre-image of this closed arc is

$$
B C^{-1}(K)=\left\{e^{i \theta} \in \mathbb{T}_{\chi}: \theta_{0} / f \leq \theta \leq \theta_{1} / f, \theta \in[0,2 \pi]\right\}
$$

which is a closed arc in $\mathbb{T}_{\chi}$. It follows that the pre-image of a compact set is compact.

(2) follows immediately from Lemma 5.8.

\section{$K$-theory}

Let $E / F$ be a finite Galois extension. The unitary dual of GL(1) is a countable disjoint union of circles and so has the structure of a locally compact Hausdorff space. The base change map

$$
B C: \sqcup \mathbb{T}_{\chi} \rightarrow \sqcup \mathbb{T}_{\eta}
$$

with $\chi \in \widehat{U_{F}}, \eta \in \widehat{U_{E}}$ is a proper map.

Each $K$-group is a countably generated free abelian group:

$$
K^{j}\left(\mathcal{A}_{1}^{t}(F)\right) \cong \bigoplus \mathbb{Z}_{\chi}, \quad K^{j}\left(\mathcal{A}_{1}^{t}(E)\right) \cong \bigoplus \mathbb{Z}_{\eta}
$$

with $\chi \in \widehat{u_{F}}, \eta \in \widehat{u_{E}}, j=0,1$, where $\mathbb{Z}_{\chi}$ and $\mathbb{Z}_{\eta}$ denote a copy of $\mathbb{Z}$.

There is a functorial map at the level of $K$-theory groups

$$
K^{j}(B C): \bigoplus \mathbb{Z}_{\eta} \rightarrow \bigoplus \mathbb{Z}_{\chi} .
$$

Base change selects among the characters of $\widehat{U_{E}}$ those of the form $\chi_{E}=\chi_{F} \circ N_{E / F}$, where $\chi_{F}$ is a character of $\widehat{U_{F}}$.

Theorem 5.10. When we restrict $K^{1}(B C)$ to the direct summand $\mathbb{Z}_{\chi_{E}}$ we get the following map:

$$
\mathbb{Z}_{\chi_{E}} \rightarrow \mathbb{Z}_{\chi_{F}}, \quad x \mapsto f \cdot x .
$$

On the remaining direct summands, $K^{1}(B C)=0$. When we restrict $K^{0}(B C)$ to the direct summand $\mathbb{Z}_{\chi_{E}}$ we get the following map:

$$
\mathbb{Z}_{\chi_{E}} \rightarrow \mathbb{Z}_{\chi_{F}}, \quad x \mapsto x .
$$

On the remaining direct summands, $K^{0}(B C)=0$. In each case, we have $c\left(\chi_{E}\right)=$ $\psi_{E / F}\left(c\left(\chi_{F}\right)\right)$. 


\section{Base change and $K$-theory for $\operatorname{GL}(2, F)$}

Through this section, $F$ denotes a nonarchimedean local field with characteristic 0 and $p \neq 2$.

Let $\mathscr{G}_{2}^{0}(F)$ be the set of equivalence classes of irreducible 2-dimensional smooth (complex) representations of $W_{F}$. Let $\mathcal{A}_{2}^{0}(F)$ be the subset of $\mathcal{A}_{2}^{t}(F)$ consisting of equivalence classes of irreducible cuspidal representations of $\operatorname{GL}(2, F)$. The local Langlands correspondence gives a bijection

$$
{ }_{F} \mathscr{L}: \mathscr{E}_{2}^{0}(F) \rightarrow \mathcal{A}_{2}^{0}(F) .
$$

We recall the concept of admissible pair [6], p. 124.

Definition 6.1. Let $E / F$ be a quadratic extension and let $\xi$ be a quasicharacter of $E^{\times}$. The pair $(E / F, \xi)$ is called admissible if

(1) $\xi$ does not factor through the norm map $N_{E / F}: E^{\times} \rightarrow F^{\times}$and,

(2) if $\xi \mid U_{E}^{1}$ does factor through $N_{E / F}$, then $E / F$ is unramified.

Denote the set of $F$-isomorphism classes of admissible pairs $(E / F, \xi)$ by $\mathcal{P}_{2}(F)$. According to [6], p. 215, the map

$$
\begin{aligned}
\mathcal{P}_{2}(F) & \rightarrow \mathscr{E}_{2}^{0}(F), \\
(E / F, \xi) & \mapsto \operatorname{Ind}_{E / F} \xi
\end{aligned}
$$

is a canonical bijection, where we see $\xi$ as a quasicharacter of $W_{E}$ via the class field theory isomorphism $W_{E}^{\mathrm{ab}} \cong E^{\times}$and $\operatorname{Ind}_{E / F}$ is the functor of induction from representations of $W_{E}$ to representations of $W_{F}$.

The tempered dual of GL(2) comprises the cuspidal representations with unitary central character, the unitary twists of the Steinberg representation, and the unitary principal series.

We will restrict ourselves to admissible pairs $(E / F, \xi)$ for which $\xi$ is a unitary character. This ensures that $\rho:=\operatorname{Ind}_{E / F} \xi$ is unitary. Therefore $\operatorname{det}(\rho)$ is unitary and $\mathscr{L}(\rho)$ has unitary central character.

The cuspidal representations of GL(2) with unitary central character arrange themselves in the tempered dual as a countable union of circles. For each circle $\mathbb{T}$, we select an admissible pair $(E / F, \xi)$ for which

$$
\mathscr{L}(\rho) \in \mathbb{T}
$$

and label this circle as $\mathbb{T}_{(E / F, \xi)}$.

We further restrict ourselves to admissible pairs $(E / F, \xi)$ for which $E / F$ is totally ramified. 
Theorem 6.2. Let $L / F$ be an unramified extension of odd degree. Then we have:

(1) Base change is a proper map.

(2) When we restrict base change to one circle we get the following:

$$
B C: \mathbb{T}_{(E / F, \xi)} \rightarrow \mathbb{T}_{\left(E L / L, \xi_{L}\right)}, \quad z \mapsto z^{f(L / F)}
$$

with $\xi_{L}=\xi \circ N_{E L / E}$ and $c\left(\xi_{L}\right)=\psi_{E L / E}(c(\xi))=c(\xi)$.

Proof. The proof of (1) is analogous to the proof of Theorem 5.9.

Each representation $\rho \in \mathscr{E}_{2}^{0}(F)$ has a torsion number: the order of the cyclic group of all the unramified characters $\chi$ for which $\chi \rho \cong \rho$. The torsion number of $\rho$ will be denoted $t(\rho)$.

Set $\sigma=\operatorname{Ind}_{E / F} \xi, \pi=\mathscr{L}(\sigma)$ and $\sigma_{L}=\operatorname{Ind}_{E L / L} \xi_{L}=\sigma \mid W_{L}$. Then $\sigma$ is totally ramified, in the sense that $t(\sigma)=1$, as in the proof of Theorem 3.3 in [5], p. 697. The pair $\left(E L / L, \xi_{L}\right)$ is admissible [5], Theorem 4.6. We now quote [5], Proposition 3.2 (7), to infer that

$$
\mathscr{L}\left(\sigma_{L}\right)=b_{L / F} \pi .
$$

If $L / F$ is unramified then, by [14], Proposition 7.2, p. 153, $E L / E$ is unramified. For the ramification indices, we have:

$$
e_{E L / F}=e_{E L / L} \times e_{L / F}=e_{E L / E} \times e_{E / F} .
$$

Since $L / F$ and $E L / E$ are both unramified it follows that

$$
e_{E L / L}=e_{E / F}=2 .
$$

Since $E L / L$ is a quadratic extension, $E L / L$ is totally ramified. Therefore $\sigma_{L}$ is totally ramified, i.e., $t\left(\sigma_{L}\right)=1$.

Hence, base change maps each circle into another circle and the map is given by $z \mapsto z^{f(L / F)}$.

Finally, since $E L / E$ is unramified, we have $\psi_{E L / E}(x)=x$ and the result follows.

Let $L / F$ be a finite unramified Galois extension. The cuspidal part of the tempered dual of GL(2) is a countable disjoint union of circles and so has the structure of a locally compact Hausdorff space. The base change map

$$
B C: \sqcup \mathbb{T}_{(E / F, \xi)} \rightarrow \sqcup \mathbb{T}_{(E L / L, \eta)}
$$

with $(E / F, \xi)$ an admissible pair, $E / F$ totally ramified and $\xi$ unitary is a proper map.

Each $K$-group is a countably generated free abelian group:

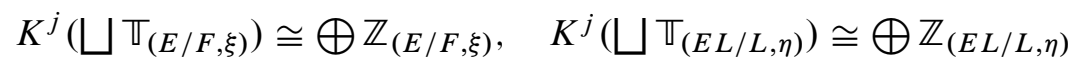


where $\mathbb{Z}_{(E / F, \xi)}$ and $\mathbb{Z}_{(E L / L, \eta)}$ denote a copy of $\mathbb{Z}, j=0,1$.

In complete analogy with GL(1) there is a functorial map at the level of $K$-theory groups

$$
K^{j}(B C): \bigoplus \mathbb{Z}_{(E L / L, \eta)} \rightarrow \bigoplus \mathbb{Z}_{(E / F, \xi)} .
$$

Base change selects among the admissible pairs $(E L / L, \eta)$ those of the form $\left(E L / L, \xi_{L}\right)$, where $\xi_{L}=\xi \circ N_{E L / E}$.

Theorem 6.3. When we restrict $K^{1}(B C)$ to the direct summand $\mathbb{Z}_{\left(E L / L, \xi_{L}\right)}$ we get the following map:

$$
\mathbb{Z}_{\left(E L / L, \xi_{L}\right)} \rightarrow \mathbb{Z}_{(E / F, \xi)}, \quad x \mapsto f(L / F) \cdot x .
$$

On the remaining direct summands, $K^{1}(B C)=0$. When we restrict $K^{0}(B C)$ to the direct summand $\mathbb{Z}_{\left(E L / L, \xi_{L}\right)}$ we get the following map:

$$
\mathbb{Z}_{\left(E L / L, \xi_{L}\right)} \rightarrow \mathbb{Z}_{(E / F, \xi)}, \quad x \mapsto x .
$$

On the remaining direct summands, $K^{0}(B C)=0$.

\section{References}

[1] J. Arthur and L. Clozel, Simple algebras, base change, and the advanced theory of the trace formula. Ann. of Math. Stud. 120, Princeton University Press, Princeton, NJ, 1989. Zbl 0682.10022 MR 1007299

[2] P. Baum, N. Higson and R. J. Plymen, A proof of the Baum-Connes conjecture for $p$ adic GL(n). C. R. Acad. Sci. Paris Sér. I Math. 325 (1997), 171-176. Zbl 0918.46061 MR 1467072

[3] A. Borel, Automorphic $L$-functions. In Automorphic forms, representations and $L$ functions (Oregon State Univ., Corvallis, Oregon, 1977), Proc. Sympos. Pure Math. 33, Part 2, Amer. Math. Soc., Providence, R.I., 1979, 27-61. Zbl 0412.10017 MR 546608

[4] J. Brodzki and R. J. Plymen, Complex structure on the smooth dual of GL(n). Doc. Math. 7 (2002), 91-112. Zbl 1012.22031 MR 1911211

[5] C. J. Bushnell and G. Henniart, The essentially tame local Langlands correspondence, I. J. Amer. Math. Soc. 18 (2005), 685-710. Zbl 1073.11070 MR 2138141

[6] C. J. Bushnell and G. Henniart, The Local Langlands Conjecture for GL(2), Grundlehren Math. Wiss. 335, Springer-Verlag, Berlin 2006. Zbl 1100.11041 MR 2234120

[7] P. Cartier, Representations of p-adic groups: a survey. In Automorphic forms, representations and L-functions (Oregon State Univ., Corvallis, Oregon, 1977), Proc. Sympos. Pure Math. 33, Part 1, Amer. Math. Soc., Providence, R.I., 1979, 111-155. Zbl 0421.22010 MR 546593

[8] I. B. Fesenko and S. V. Vostokov, Local fields and their extensions. Transl. Math. Monogr. 121, 2nd ed., Amer. Math. Soc., Providence, R.I., 2002. Zbl 01793794 MR 1915966 
[9] G. Henniart, Une preuve simple des conjectures de Langlands pour GL $(n)$ sur un corps p-adique. Invent. Math. 139 (2000), 439-455. Zbl 1048.11092 MR 1738446

[10] M. Harris and R. Taylor, The geometry and cohomology of some simple Shimura varieties. Ann. of Math. Stud. 151, Princeton University Press, Princeton, NJ, 2001. Zbl 1036.11027 MR 1876802

[11] V. Lafforgue, $K$-théorie bivariante pour les algèbres de Banach et conjecture de BaumConnes. Invent. Math. 149 (2002), 1-95. Zbl 1084.19003 MR 1914617

[12] G. Laumon, M. Rapoport and U. Stuhler, D-elliptic sheaves and the Langlands correspondence. Invent. Math. 113 (1993), 217-338. Zbl 0809.11032 MR 1228127

[13] Yu. I. Manin and A. A. Panchishkin, Introduction to modern number theory. Encyclopaedia Math. Sci. 49, Springer-Verlag, Berlin 2005. Zbl 1079.11002 MR 2153714

[14] J. Neukirch, Algebraic number theory. Grundlehren Math. Wiss. 322, Springer-Verlag, Berlin 1999. Zbl 0956.11021 MR 1697859

[15] R. J. Plymen, The reduced $C^{*}$-algebra of the $p$-adic group GL(n). J. Funct. Anal. 72 (1987), 1-12. Zbl 0625.46064 MR 883498

[16] J.-P. Serre, Local fields. Grad. Texts in Math. 67, Springer-Verlag, New York 1979. Zbl 0423.12016 MR 554237

[17] J. Tate, Number theoretic background. In Automorphic forms, representations and Lfunctions (Oregon State Univ., Corvallis, Oregon, 1977), Proc. Sympos. Pure Math. 33, Part 2, Amer. Math. Soc., Providence, R.I., 1979, 3-26. Zbl 0422.12007 MR 546607

[18] A. Weil, Basic number theory. Classics Math., Springer-Verlag, Berlin 1995. Zbl 0823.11001 MR 1344916

Received June 6, 2006; revised February 15, 2007

S. Mendes, ISCTE, Av. das Forças Armadas, 1649-026, Lisbon, Portugal

E-mail: sergio.mendes@iscte.pt

R. Plymen, School of Mathematics, Manchester University, Manchester M13 9PL, England

E-mail: plymen@manchester.ac.uk 